Article

\title{
Stability of Anthocyanins and Their Degradation Products from Cabernet Sauvignon Red Wine under Gastrointestinal $\mathrm{pH}$ and Temperature Conditions
}

\author{
Ping Yang ${ }^{1,+}$, Chunlong Yuan ${ }^{1,2,3,+}$, Hua Wang ${ }^{1,2,3, *}$, Fuliang Han ${ }^{1,2,3, *}$, Yangjie Liu ${ }^{1}$, \\ Lin Wang ${ }^{1}$ and Yang Liu ${ }^{1}$ \\ 1 College of Enology, Northwest A\&F University, Yangling 712100, China; duolamiwawa@126.com (P.Y.); \\ yuanchunlong@hotmail.com (C.Y.); j1349266163@163.com (Y.L.); maggie_wongkaixin@163.com (L.W.); \\ pjxy122ly@163.com (Y.L.) \\ 2 Shaanxi Engineering Research Center for Viti-Viniculture, Northwest A\&F University, \\ Yangling 712100, China \\ 3 Heyang Viticulture Experimental Station, Northwest A\&F University, Heyang 715300, China \\ * Correspondence: wanghua@nwsuaf.edu.cn (H.W.); hanfl@nwsuaf.edu.cn (F.H.); \\ Tel.: +86-29-8709-2107 (H.W. \& F.H.) \\ + These authors contributed equally to this work.
}

Received: 14 January 2018; Accepted: 5 February 2018; Published: 7 February 2018

\begin{abstract}
This study investigated the stability of wine anthocyanins under simulated gastrointestinal $\mathrm{pH}$ and temperature conditions, and further studied the evolution of anthocyanin degradation products through simulated digestive conditions. The aim of this study was to investigate the relation between anthocyanins' structure and their digestive stability. Results showed that a total of 22 anthocyanins were identified in wine and most of these anthocyanins remained stable under simulated gastric digestion process. However, a dramatic concentration decrease happened to these anthocyanins during simulated intestinal digestion. The stability of anthocyanins in digestive process appeared to be related to their structure. The methoxy group in the B-ring enhanced the stability of anthocyanins, whereas hydroxyl group resulted in a reduction of their stability. Acylation decreased the stability of malvidin 3-O-glucoside. Pyruvic acid conjugation enhanced the structural stability of pyranoanthocyanins, whereas acetaldehyde attachment weakened their stability. A commercial malvidin 3-O-glucoside standard was used to investigate anthocyanin degradation products under simulated digestion process, and syringic acid, protocatechuic acid and vanillic acid were confirmed to be the degradation products via anthocyanin chalcone conversion path. Gallic acid, protocatechuic acid, vanillic acid, syringic acid, and $p$-coumaric acid in wine experienced a significant concentration decrease during digestion process. However, wine model solution revealed that phenolic acids remained stable under gastrointestinal conditions, except gallic acid.
\end{abstract}

Keywords: Cabernet Sauvignon wine; anthocyanins; degradation; stability; gastrointestinal $\mathrm{pH}$ and temperature

\section{Introduction}

Anthocyanins are the major colorants in grapes and wine, and they have been confirmed to play important roles in affecting the appearance and sensory attributes of grapes and wine [1-5]. More importantly, anthocyanins can serve as one of the most important antioxidants in grapes and wine to provide multiple health promoting properties [6-8]. For example, it has been reported that anthocyanins possess the anti-cancer, anti-inflammatory, antimicrobial features, and the consumption of these antioxidants can lower the incidence of cardiovascular, diabetic, and obesity diseases [9-15]. 
Anthocyanins can be synthesized as secondary metabolites in grapes during the grape development period [16,17]. These colorants can be extracted into red wine during the wine maceration process and they can further be metabolized during the wine fermentation and aging period $[1,18,19]$. Therefore, the composition and concentration of anthocyanins mainly determine the appearance of red wine. It has been reported that the level of anthocyanins in red wine ranges from 10 to $2000 \mathrm{mg} / \mathrm{L}$ [19-21], and their level in wine is mainly determined by grape variety, climate conditions, management system, and fermentation technique [3,19,22-26]. Regarding their chemical nature, anthocyanins can be divided into monomeric anthocyanins (Figure 1), acylated anthocyanins, pyranoanthocyanins, and polymeric anthocyanins [27-31]. In Vitis vinifera grapes and wine, the major monomeric anthocyanins include pelargonidin 3-O-glucoside, delphinidin 3-O-glucoside, cyanidin 3-O-glucoside, petunidin 3-O-glucoside, peonidin 3-O-glucoside, and malvidin 3-O-glucoside (Figure 1) [2,26,31]. The monomeric anthocyanins can be further converted into the acetylated, coumaroylated, and caffeoylated anthocyanins [2,30,31]. Pyranoanthocyanins can be synthesized through conjugating monomeric or acylated anthocyanins with low molecular compounds in wine, whereas the interaction among anthocyanins or between anthocyanins and other phenolic compounds could result in the formation of polymeric anthocyanins [30-33]. Pyranoanthocyanins and polymeric anthocyanins normally are formed during wine aging process, which could shift wine color from bright red color to brick and dark red color [34,35].

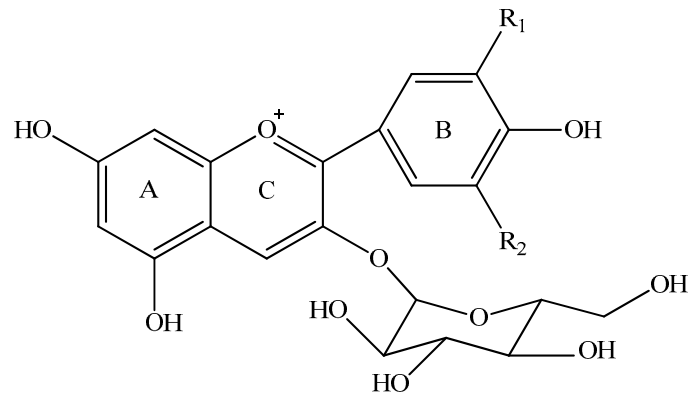

\begin{tabular}{ccc}
\hline Anthocyanins & $\mathbf{R}_{\mathbf{1}}$ & $\mathbf{R}_{\mathbf{2}}$ \\
\hline Delphinidin 3-O-glucoside & $\mathrm{OH}$ & $\mathrm{OH}$ \\
Cyanidin 3-O-glucoside & $\mathrm{OH}$ & $\mathrm{H}$ \\
Petunidin 3-O-glucoside & $\mathrm{OCH}_{3}$ & $\mathrm{OH}$ \\
Peonidin 3-O-glucoside & $\mathrm{OCH}_{3}$ & $\mathrm{H}$ \\
Malvidin 3-O-glucoside & $\mathrm{OCH}_{3}$ & $\mathrm{OCH}_{3}$ \\
\hline
\end{tabular}

Figure 1. Five monomeric anthocyanins in red wine of Vitis Vinifera L.

It has been reported that anthocyanins, like other phenolic compounds, can be absorbed through the human gastrointestinal tract. The absorption rate of anthocyanins is reported to be around $0.1 \%$ to $2 \%$, and they could be readily distributed to different organs, including liver, lung, kidney, prostate, heart, and brain [6,36-38]. The major digestive segments in the human gastrointestinal tract include stomach and small intestine. Nutrient digestion mainly happens in the stomach, whereas the small intestine is the major place where nutrients are absorbed [39,40]. It has been reported that $10 \%$ to $25 \%$ of anthocyanins were degraded in stomach, whereas the small intestine was responsible for $30 \%$ to $50 \%$ of the anthocyanins' degradation [41-45]. Anthocyanin degradation can happen at their A- and/or B-ring. The A-ring degradation has been reported to result in the formation of formylphloroglucinal or phloroglucinol carboxylic acid, whereas syringic, vanillic and protocatechuic acids were the major products from the anthocyanins degradation via B-ring cleavage [46-48].

It has been accepted that the percentage of anthocyanins' degradation in the human gastrointestinal tract is closely correlated to the anthocyanins' structure. However, such investigations have not been well documented to our best knowledge, especially regarding the acylated anthocyanins, 
pyranoanthocyanins, and polymeric anthocyanins found in wine. Therefore, the present study selected anthocyanins in Cabernet Sauvignon wine, and further investigated their stability under simulated human gastrointestinal $\mathrm{pH}$ and temperature conditions. Furthermore, anthocyanin standards and their potential degradation products were also investigated under the simulated gastrointestinal conditions to elucidate the degradation pathway of anthocyanins. The findings from this study could provide useful information on the elucidation of the anthocyanins' stability and metabolism in the human gastrointestinal tract.

\section{Results and Discussion}

\subsection{Anthocyanins and Phenolic Acids in Red Wine}

A total of 22 anthocyanins were identified in the wine samples, including five monomeric anthocyanins, six acylated anthocyanins, eight pyranoanthocyanins, and three polymeric anthocyanins (Figure 2a and Table 1).

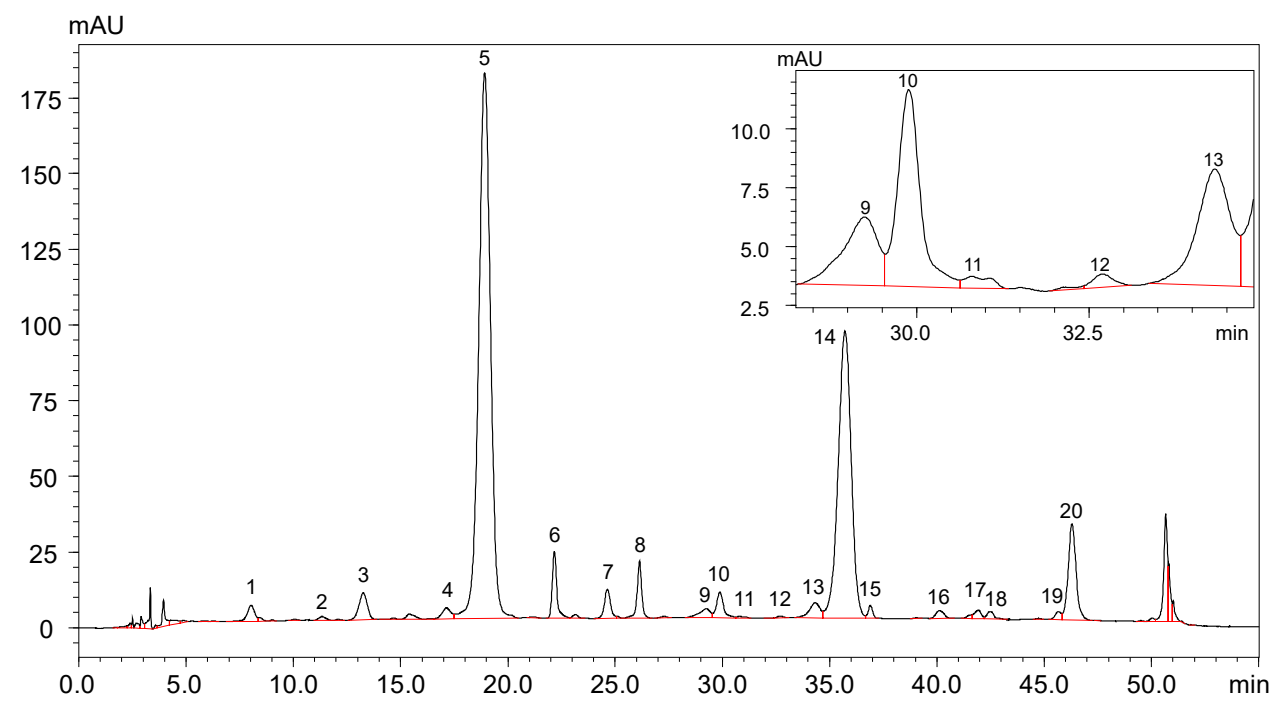

(a)

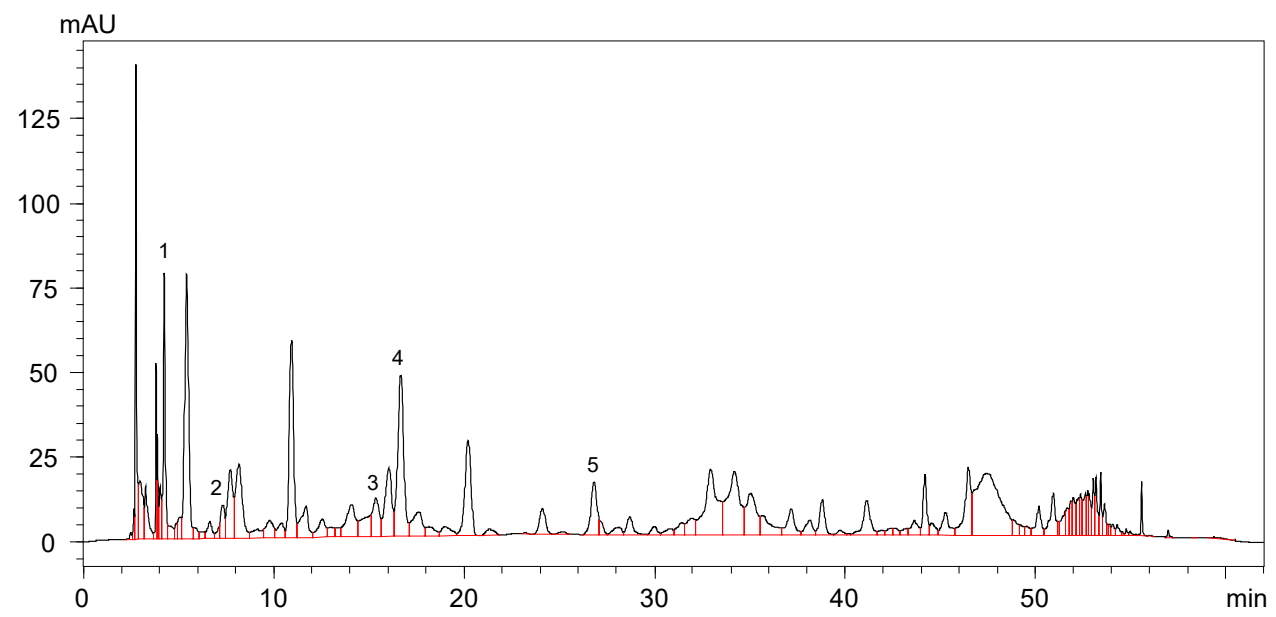

(b)

Figure 2. HPLC chromatography of (a) identified anthocyanins and (b) phenolic acids in Cabernet Sauvignon red wine. 
Table 1. Anthocyanins found in Cabernet Sauvignon red wine.

\begin{tabular}{|c|c|c|c|c|c|c|}
\hline Peak No. & Compounds & Rt (min) & $\lambda \max$ & Precursor Ion & Product Ion & Concentration $(\mathrm{mg} / \mathrm{L})$ \\
\hline 1 & Delphinidin 3-O-glucoside & 7.978 & 524 & 465 & 303 & $2.801 \pm 0.011$ \\
\hline 2 & Cyanidin 3-O-glucoside & 11.297 & 524 & 449 & 287 & $0.814 \pm 0.010$ \\
\hline 3 & Petunidin 3-O-glucoside & 13.226 & 524 & 479 & 317 & $5.047 \pm 0.014$ \\
\hline 4 & Peonidin 3-O-glucoside & 17.137 & 523 & 463 & 301 & $2.295 \pm 0.001$ \\
\hline 5 & Malvidin 3-O-glucoside & 18.921 & 276,525 & 493 & 331 & $137.815 \pm 0.396$ \\
\hline 6 & Vitisin A & 22.183 & 510 & 561 & 399 & $6.743 \pm 0.015$ \\
\hline 7 & Vitisin B & 24.669 & - & 517 & 355 & $4.106 \pm 0.032$ \\
\hline 8 & Malvidin 3-O-(6-O-acetyl)-glucoside-pyruvic acid & 26.168 & 514 & 603 & 399 & $5.916 \pm 0.026$ \\
\hline 9 & Malvidin 3-O-glucoside-ethyl-catechin (1) & 29.29 & 525 & 809 & 357 & $1.999 \pm 0.357$ \\
\hline 10 & Malvidin 3-O-(6-O-acetyl)-glucoside-acetaldehyde & 29.926 & 495 & 559 & 355 & $4.012 \pm 0.032$ \\
\hline 11 & Malvidin 3-O-glucoside-ethyl-catechin (2) & 30.847 & 532 & 809 & 357 & $0.337 \pm 0.008$ \\
\hline 12 & Malvidin 3-O-glucoside-ethyl-catechin (3) & 32.762 & 532 & 809 & 357 & $0.404 \pm 0.008$ \\
\hline 13 & Peonidin 3-O-(6-O-acetyl)-glucoside & 34.396 & 523 & 505 & 301 & $3.530 \pm 0.464$ \\
\hline 14 & Malvidin 3-O-(6-O-acetyl)-glucoside & 35.799 & 528 & 535 & 331 & $80.068 \pm 0.419$ \\
\hline 15 & Malvidin 3-O-(6-O-p-coumaryl)-glucoside-pyruvic acid & 36.989 & 517 & 707 & 399 & $1.382 \pm 0.018$ \\
\hline 16 & Malvidin 3-O-(6-O-caffeoyl)-glucoside & 40.239 & 531 & 655 & 331 & $1.510 \pm 0.382$ \\
\hline 17 & Malvidin 3-O-(6-O-p-coumaryl)-glucoside-acetaldehyde & 42.046 & - & 663 & 355 & $1.624 \pm 0.208$ \\
\hline 18 & Malvidin 3-O-(6-O-cis-p-coumaryl)-glucoside & 42.615 & 532 & 639 & 331 & $1.354 \pm 0.034$ \\
\hline 19 & Peonidin 3-O-(6-O-trans- $p$-coumaryl)-glucoside & 45.814 & 524 & 609 & 301 & $1.175 \pm 0.433$ \\
\hline 20 & Malvidin 3-O-(6-O-trans- -coumaryl)-glucoside & 46.45 & 531 & 639 & 331 & $16.081 \pm 0.570$ \\
\hline 21 & Malvidin 3-O-glucoside-4-vinylphenol adduct & - & - & 609 & 447 & - \\
\hline \multirow[t]{2}{*}{22} & Malvidin 3-O-(6-O-acetyl)-glucoside-4-vinylphenol adduct & - & - & 651 & 447 & - \\
\hline & Total anthocyanins & - & - & - & - & $299.752 \pm 2.352$ \\
\hline
\end{tabular}


It should be noted that malvidin 3-O-glucoside-4-vinylphenol adduct and malvidin 3-O-(6-Oacetyl)-glucoside-4-vinylphenol adduct were not quantified due to their limited concentration in the wine sample. The total anthocyanins concentration in the wine sample was about $299.75 \mathrm{mg} / \mathrm{L}$ (Table 1). Malvidin 3-O-glucoside appeared to be the highest individual anthocyanin in the wine sample and its concentration represented more than $49 \%$ of the total anthocyanin concentration. Besides, malvidin 3-O-(6-O-acetyl)-glucoside and malvidin 3-O-(6-O-trans-p-coumaryl)-glucoside were also found to be the predominant anthocyanins in the wine sample, and their concentration accounted for about $29 \%$ and $6 \%$ of the total anthocyanins content, respectively. Gallic acid, protocatechuic acid, vanillic acid, syringic acid, and $p$-coumaric acid were all detected in the wine sample (Figure $2 \mathrm{~b}$ and Table 2). It should be noted that syringic acid exhibited the highest concentration in the wine sample, whereas $p$-coumaric acid existed in the wine with the lowest concentration.

Table 2. Phenolic acids found in Cabernet Sauvignon red wine.

\begin{tabular}{ccccc}
\hline Peak No. & Compounds & Rt $(\mathbf{m i n})$ & $\lambda \max$ & Concentration $(\mathbf{m g} / \mathrm{L})$ \\
\hline 1 & Gallic acid & 4.243 & 271 & $11.676 \pm 0.321$ \\
2 & Protocatechuic acid & 7.319 & 259,293 & $4.771 \pm 0.208$ \\
3 & Vanillic acid & 15.363 & 291 & $8.156 \pm 0.853$ \\
4 & Syringic acid & 16.671 & 274 & $21.350 \pm 0.412$ \\
5 & $p$-Coumaric acid & 26.818 & 309 & $2.846 \pm 0.162$ \\
\hline
\end{tabular}

\subsection{Evolution of Anthocyanins and Phenolic Acids under Simulated Gastrointestinal pH and} Temperature Conditions

\subsubsection{Total Anthocyanins}

Figure 3 shows the evolution of the total wine anthocyanins in the simulated gastrointestinal $\mathrm{pH}$ and temperature conditions. It was observed that incubating the wine sample in the simulated gastric condition significantly resulted in an apparent $8.22 \%$ increase of the total anthocyanin concentration after $6 \mathrm{~h}(p<0.05)$. However, its concentration decreased significantly by about $49 \%$ after digesting the wine in the simulated intestinal $\mathrm{pH}$ condition $($ after $12 \mathrm{~h})(p<0.05)$. Our result was consistent with the previous report [44].

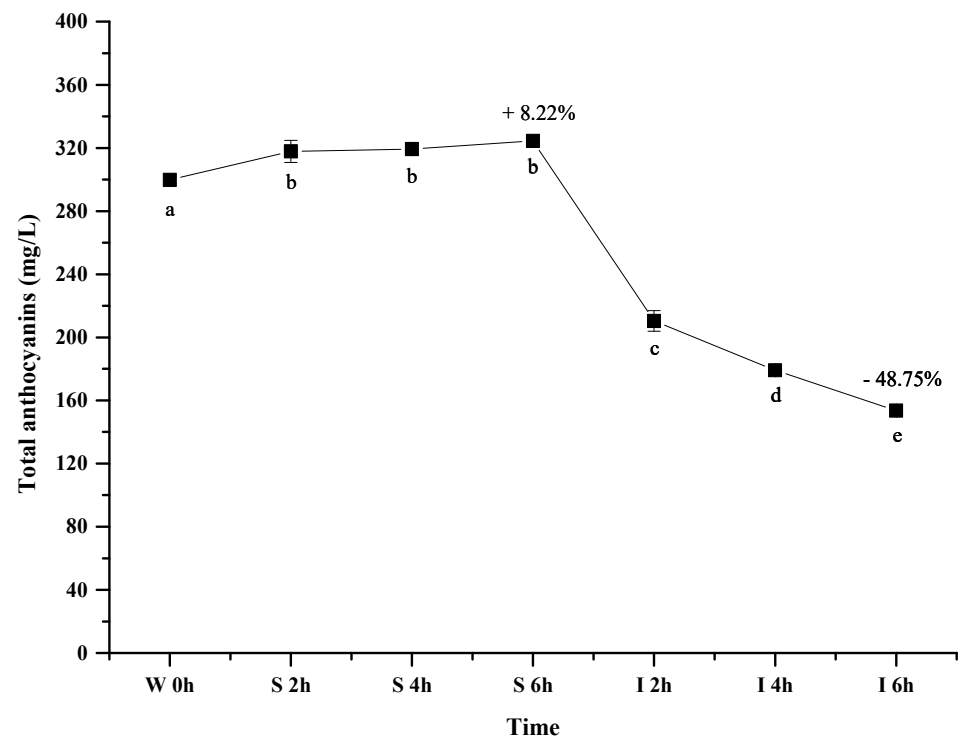

Figure 3. Content alteration of total anthocyanins in red wine during simulated digestion process (wine sample: W 0 h; S 2 h, S 4 h, S 6 h: samples under the gastric condition; I 2 h, I 4 h, I 6 h: samples under the intestinal condition; different lowercase letter means significant difference at 0.05 level). 
It has been known that anthocyanin molecules possess an equilibrium under different $\mathrm{pH}$ conditions, and lower $\mathrm{pH}$ environment could result most anthocyanins in the flavylium cation conformation [49]. Such a conformation shift led to an apparent increase on the total anthocyanins concentration under the simulated gastric condition. In the simulated intestinal tract, the decrease of the total anthocyanins concentration mainly resulted from the degradation of anthocyanins via cleaving their aromatic ring as suggested from previously studies [47,50]. Additionally, basic condition in the simulated intestinal tract was also reported to trigger the interactions between anthocyanin molecules and other compounds to yield polymeric compounds [43,51]. Such interactions could also result in the decrease of the total anthocyanins concentration.

\subsubsection{Monomeric Anthocyanins}

Five monomeric anthocyanins (Figure 1), including malvidin 3-O-glucoside, petunidin 3-O-glucoside, peonidin 3-O-glucoside, delphinidin 3-O-glucoside, and cyanidin 3-O-glucoside, were detected in the wine sample. Malvidin 3-O-glucoside, petunidin 3-O-glucoside, peonidin 3-O-glucoside, and delphinidin 3-O-glucoside exhibited the similar evolution pattern under the simulated gastrointestinal conditions (Figure 4). These four monomeric anthocyanins increased their apparent concentration in the simulated gastric condition, and then significantly decreased their level under the simulated intestinal tract $(p<0.05)$. Our results were in accordance with the previous reports $[44,45,52]$. Flavylium cation conformation has been confirmed to be the dominant structure for monomeric anthocyanins under the acidic condition, and this conformation can benefit the stability of anthocyanins [49]. This could explain the apparent increase on these monomeric anthocyanins during the incubation at the simulated gastric tract. The basic condition in the simulated intestinal condition could facilitate the cleavage of the C-ring in anthocyanins structure, which led to a concentration decrease of these monomeric anthocyanins [42]. It should be worth noting that cyanidin 3-O-glucoside exhibited an opposite evolution during the incubation at the simulated gastrointestinal condition compared to the other monomeric anthocyanins. A significant concentration decrease of cyanidin 3-O-glucoside was found under the simulated gastric conditions, whereas incubating the wine under the simulated intestinal condition resulted in a concentration increase and then a decrease of this anthocyanin $(p<0.05)$ (Figure 4$)$. Acidic conditions might cause the release of the sugar moiety from its aglycone due to hydrolysis, whereas flavylium cation conformation could stabilize anthocyanins under acidic conditions [40]. Therefore, we speculated that hydrolysis and/or polymeric reaction might take place more rapidly on cyanidin 3-O-glucoside than the formation of its flavylium cation structure, which caused its concentration decrease under the simulated gastric tract [43,51]. During the incubation at the simulated intestinal condition, cyanidin 3-O-glucoside exhibited a concentration increase initially, followed by a decrease. Its concentration increase might result from the degradation of polymeric anthocyanins or bound anthocyanins or other unknown reason [51,53]. However, the basic condition eventually caused cyanidin 3-O-glucoside to be degraded, which lowered its concentration in the simulated intestinal condition.

It should be noted that after the digestion process the concentrations of delphinidin 3-O-glucoside, petunidin 3-O-glucoside, malvidin 3-O-glucoside, peonidin 3-O-glucoside, and cyanidin 3-O-glucoside decreased significantly by $92.9 \%, 84.3 \%, 51.1 \%, 49.7 \%$ and $46.7 \%$, respectively $(p<0.05)$ (Figure 4 ). Their stability in the digestive system appeared to be related to their molecular structure. The increase on the substituent groups in the B-ring of anthocyanins resulted in a significant decrease on the stability of the monomeric anthocyanins in the simulated gastrointestinal tract, whereas the methoxy group presence on the B-ring could stabilize the anthocyanins structure in the digestive system $[54,55]$. It should be aware that more hydroxyl groups in the anthocyanins structure accelerated the degradation of anthocyanins in the digestive system [54,55]. 


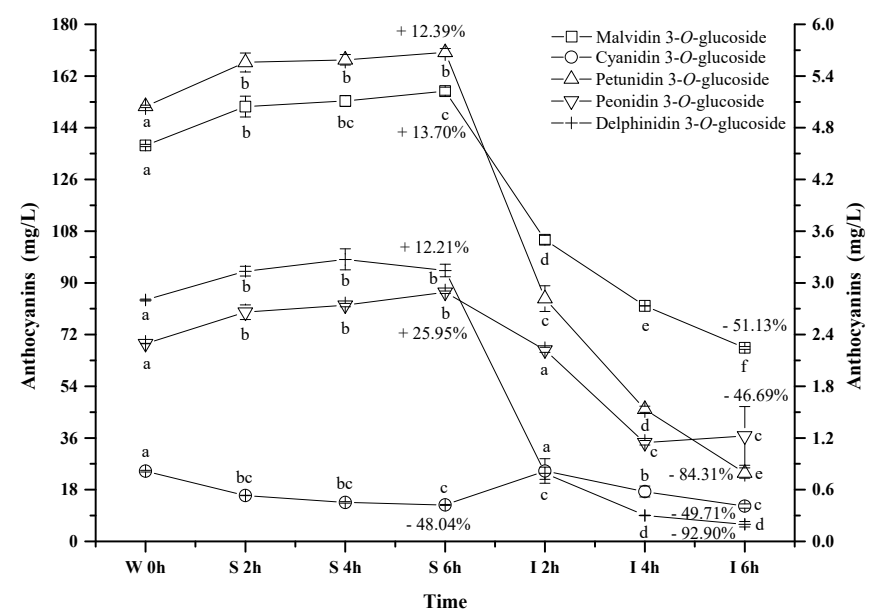

Figure 4. Content alteration of monomeric anthocyanins in red wine during simulated digestion process (Left axis: malvidin 3-O-glucoside; right axis: other anthocyanins; wine sample: $\mathrm{W} 0 \mathrm{~h}$; $\mathrm{S} 2 \mathrm{~h}$, S 4 h, S 6 h: samples under the gastric condition; I 2 h, I 4 h, I 6 h: samples under the intestinal condition; different lowercase letter means significant difference at 0.05 level).

\subsubsection{Acylated Anthocyanins}

Peonidin 3-O-(6-O-trans-p-coumaryl)-glucoside exhibited an apparent concentration increase (about $7.62 \%$ ) under the simulated gastric conditions, whereas the simulated intestinal conditions reduced its concentration by $26.27 \%(p<0.05)$ (Figure $5 \mathrm{a})$. Its evolution pattern was similar to that of peonidin 3-O-glucoside under the digestive conditions. However, a continuous concentration decrease happened to peonidin 3-O-(6-O-acetyl)-glucoside during the whole digestion process $(p<0.05)$. For instance, about a $7.3 \%$ concentration decrease was observed under the simulated gastric conditions, whereas its concentration decreased by $68.58 \%$ after the incubation under the simulated intestinal conditions. These indicated that the presence of an acetyl group in the peonidin 3-O-glucoside structure might lower the structural stability under acidic and basic conditions. In addition, peonidin 3-O-(6-O-acetyl)-glucoside or its chalcone structure might react with other compounds (such as acetaldehyde, pyruvic acid, etc.) to form the new unknown compounds [43,51]. Compared to the acetyl group, the coumaryl group addition stabilized the structure of peonidin 3-O-glucoside, leading to a lesser degradation rate under the simulated intestinal conditions.

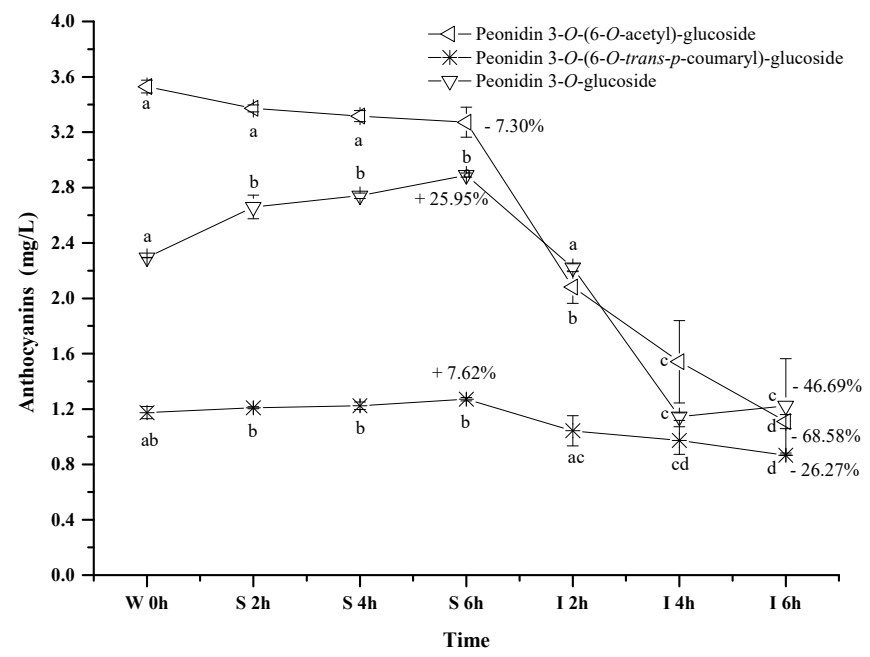

(a)

Figure 5. Cont. 


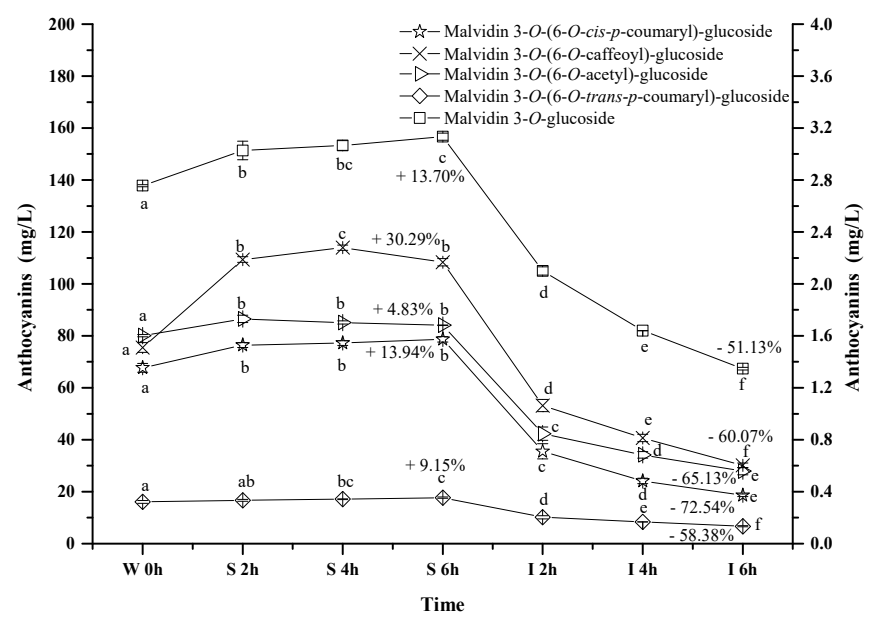

(b)

Figure 5. Content alteration of acylated anthocyanins in red wine during simulated digestion process (Left axis: malvidin 3-O-(6-O-acetyl)-glucoside, malvidin 3-O-(6-O-trans- $p$-coumaryl)-glucoside, malvidin 3-O-glucoside; Right axis: malvidin 3-O-(6-O-cis-p-coumaryl)-glucoside, malvidin 3-O-(6-O-caffeoyl)-glucoside; wine sample: $\mathrm{W} 0 \mathrm{~h}$; S 2 h, S 4 h, S 6 h: samples under the gastric condition; I 2 h, I 4 h, I 6 h: samples under the intestinal condition; different lowercase letter means significant difference at 0.05 level).

Similarly, the concentrations of malvidin 3-O-(6-O-acetyl)-glucoside, malvidin 3-O-(6-Otrans-p-coumaryl)-glucoside, malvidin 3-O-(6-O-cis-p-coumaryl)-glucoside, and malvidin 3-O-(6-Ocaffeoyl)-glucoside apparently increased by $4.83 \%, 9.15 \%, 13.94 \%$, and $30.29 \%$ after $6 \mathrm{~h}$ incubation under the simulated gastric conditions, respectively $(p<0.05)$ (Figure $5 b)$. However, their concentration dramatically decreased by $65.13 \%, 58.38 \%, 72.45 \%$ and $60.07 \%$ under the simulated intestinal conditions, respectively $(p<0.05)$. These results indicated that different substituent groups affected the stability of anthocyanins in the simulated digestive system, and the trans-p-coumaryl addition exhibited the best enhancement on the stability of anthocyanin structure, followed by the caffeoyl group, acetyl group, and the least, the cis-p-coumaryl group.

\subsubsection{Pyranoanthocyanins}

The pyranoanthocyanins formed in the wine sample of the present study mainly resulted from the interactions through malvidin 3-O-glucoside or its acylated anthocyanins with pyruvic acid (Figure 6a). For example, vitisin A is synthesized by conjugating malvidin 3-O-glucoside with pyruvic acid. This pyranoanthocyanin significantly increased its apparent concentration in the simulated gastric condition $(p<0.05)$. Acetylvitisin A and coumarylvitisin A after the incubation in the simulated gastric condition remained at similar concentrations, however, their concentration decreased by $17.73 \%, 19.77 \%$ and $27.15 \%$ after the incubation under the simulated intestinal conditions, respectively $(p<0.05)$. Compared to the evolution of malvidin 3-O-glucoside during the simulated digestion process, the presence of pyruvic acid in the malvidin 3-O-glucoside structure enhanced its stability. However, this structural addition reduced the stability of malvidin 3-O-(6-O-acetyl)-glucoside and malvidin 3-O-(6-O-p-coumaryl)-glucoside under the simulated digestive conditions. Additionally, vitisin $\mathrm{B}$ and malvidin 3-O-(6-O-p-coumaryl)-glucoside-acetaldehyde exhibited an apparent concentration increase under the simulated gastric conditions, whereas a concentration decrease was observed in malvidin 3-O-(6-O-acetyl)-glucoside-acetaldehyde $(p<0.05)$ (Figure $6 \mathrm{~b})$. These pyranoanthocyanins experienced a dramatic concentration decrease $(61.30 \%, 79.08 \%$, and $75.31 \%$, respectively) under the simulated intestinal conditions $(p<0.05)$. These indicated that acetaldehyde conjugation weakened the stability of vitisin $B$ and it derivatives. It should be noted that vitisin $A$ and its derivatives were found to be more stable under the simulated digestive condition compared to vitisin $\mathrm{B}$ and its derivatives. 


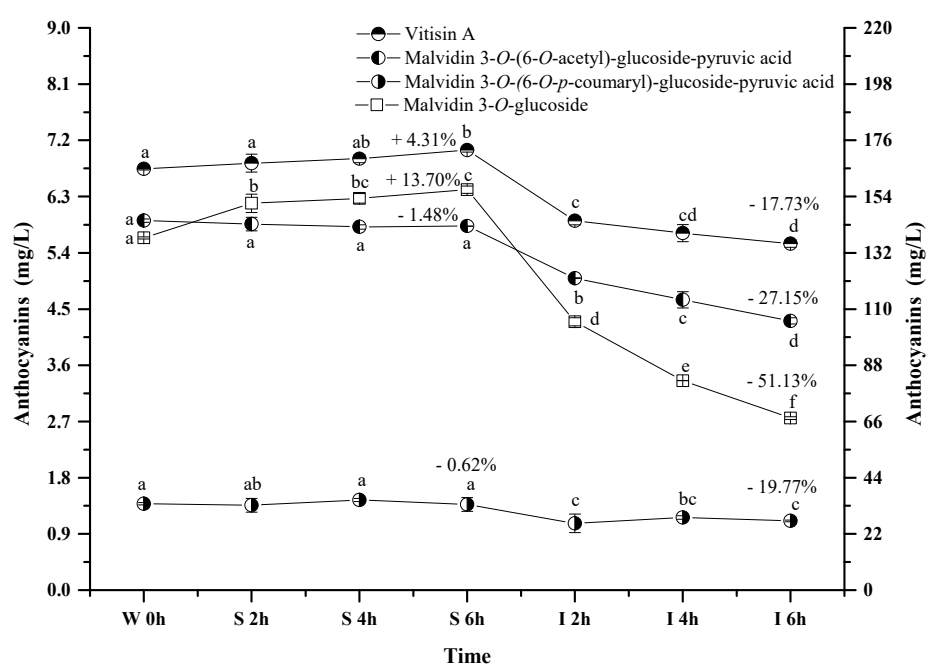

(a)

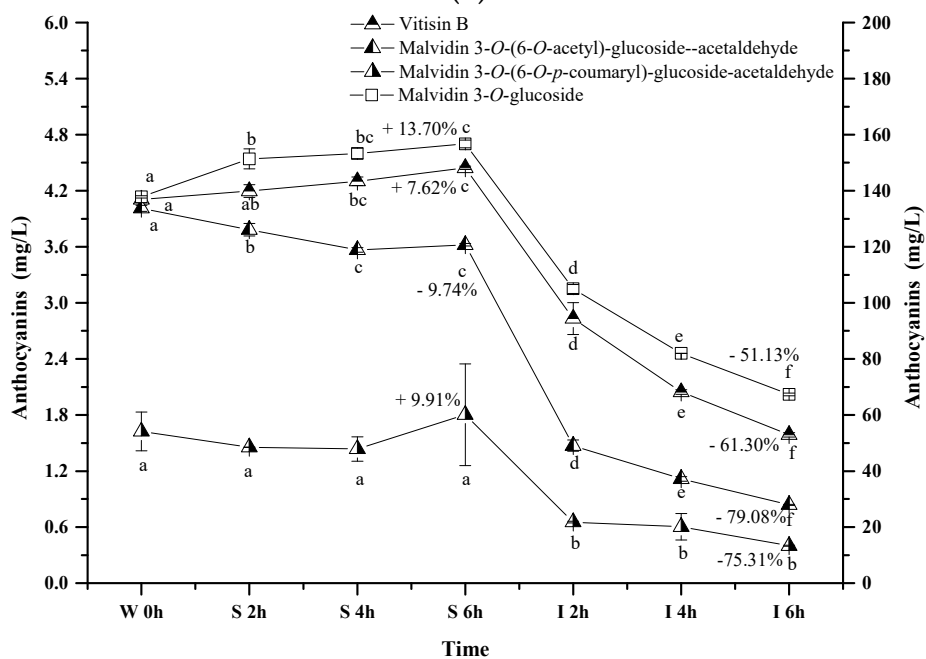

(b)

Figure 6. Content alteration of pyranoanthocyanins in red wine during simulated digestion process (wine sample: W 0 h; S 2 h, S 4 h, S 6 h: samples under the gastric condition; I 2 h, I 4 h, I 6 h: samples under the intestinal condition; different lowercase letter means significant difference at 0.05 level).

\subsubsection{Polymeric Anthocyanins}

Only three polymeric anthocyanins, including malvidin 3-O-glucoside-ethyl-catechin (1), malvidin 3-O-glucoside-ethyl-catechin (2), and malvidin 3-O-glucoside-ethyl-catechin (3), were tentatively identified in the wine sample. These polymeric anthocyanins exhibited the different evolution patterns in the simulated digestive condition (Figure 7). Malvidin 3-O-glucosideethyl-catechin (1) showed a decreasing trend on its concentration, whereas an increase happened to malvidin 3-O-glucoside-ethyl-catechin (3) $(p<0.05)$. Malvidin 3-O-glucoside-ethyl-catechin (2) showed a concentration increase under the simulated gastric condition but a concentration decrease in the simulated intestinal environment $(p<0.05)$. Under acidic conditions, the concentration increase might result from the reaction between malvidin 3-O-glucoside and catechin, whereas the concentration decrease might result from the further reaction between malvidin 3-O-glucoside-ethyl-catechin and other compounds in red wine $[56,57]$. Under alkaline conditions, the concentration variation might be due to their degradations or the degradations of other bigger molecular polymeric anthocyanins $[56,57]$. The mechanism behind these polymeric anthocyanins evolution needs to be further investigated. 


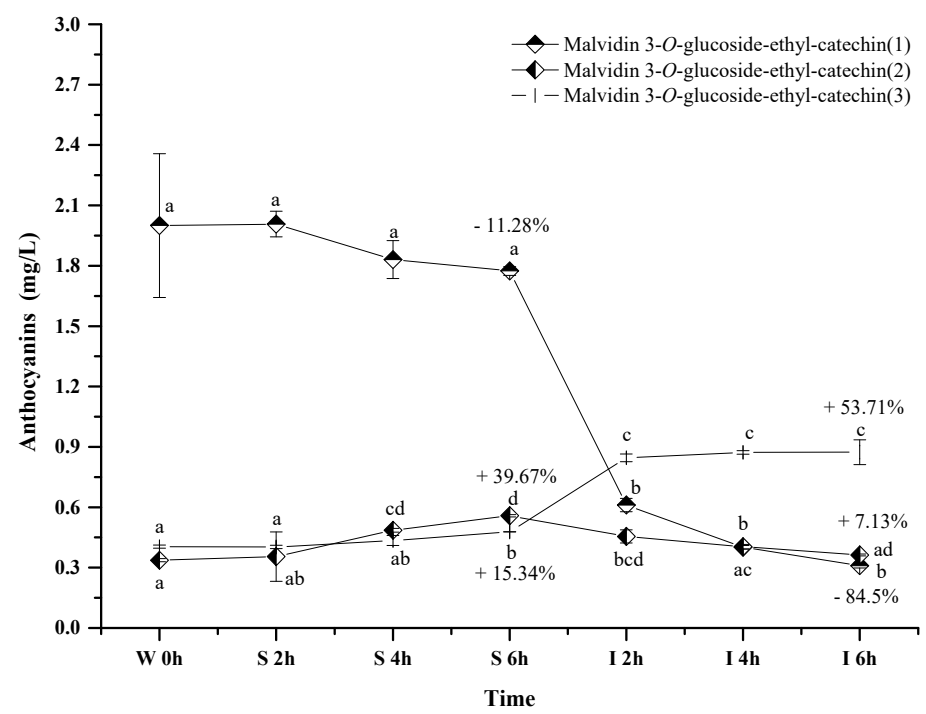

Figure 7. Content alteration of polymeric anthocyanins in red wine during simulated digestion process (wine sample: W 0 h; S 2 h, S 4 h, S 6 h: samples under the gastric condition; I 2 h, I 4 h, I 6 h: samples under the intestinal condition; different lowercase letter means significant difference at 0.05 level).

\subsubsection{Phenolic Acids}

It has been reported that anthocyanins can be degraded into phenolic acids during the digestion process, and gallic acid, protocatechuic acid, vanillic acid, syringic acid and $p$-coumaric acid have been reported to be the major anthocyanins degradation products $[46,47,58]$. Therefore, we investigated the evolution of these phenolic acids from the wine under the simulated digestion process (Figure 8).

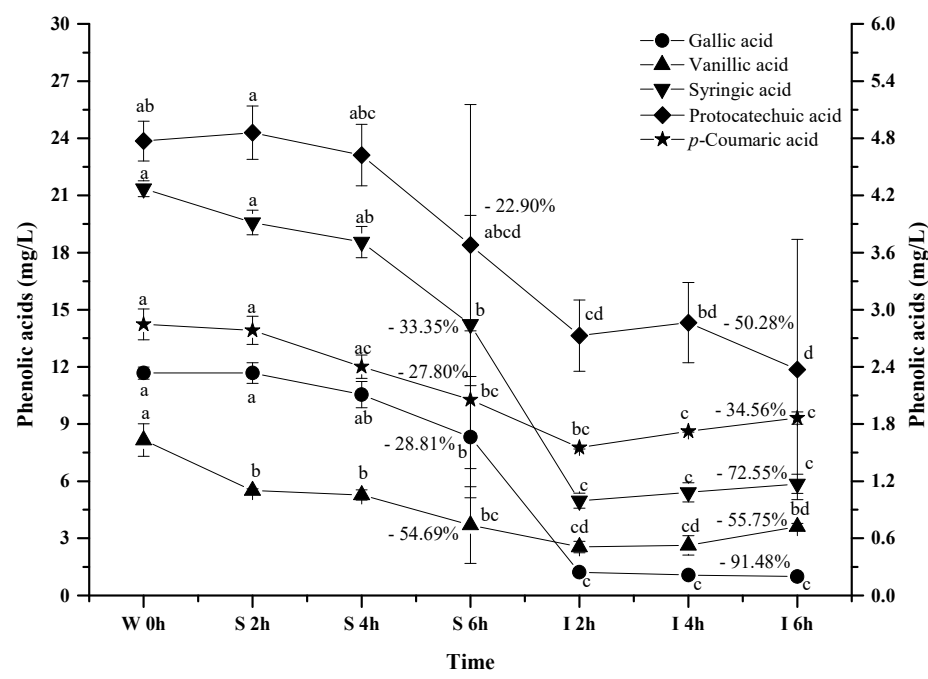

Figure 8. Content alteration of phenolic acids in red wine during simulated digestion process (wine sample: W 0 h; S 2 h, S 4 h, S 6 h: samples under the gastric condition; I 2 h, I 4 h, I 6 h: samples under the intestinal condition; different lowercase letter means significant difference at 0.05 level).

As the simulated digestion proceeded, an increasing trend on these phenolic acids was expected since most anthocyanins exhibited a dramatic concentration decrease. However, a continuous concentration decrease took place for all the phenolic acids during the digestion process $(p<0.05)$. For example, these phenolic acids decreased their concentration by $28.81 \%, 22.92 \%, 54.69 \%, 33.35 \%$, and $27.80 \%$ after the simulated gastric digestion process, respectively $(p<0.05)$. Furthermore, the incubation under the simulated intestinal condition continued to decrease their concentration by 
$91.4 \%, 50.28 \%, 55.75 \%, 72.55 \%$, and $34.56 \%(p<0.05)$. These indicated that these phenolic acids were more stable under acidic conditions than the basic conditions. More importantly, these indicated that the degradation/transformation rate of these phenolic acids was faster than their rate of formation from the anthocyanins' degradation under the simulated digestion process.

\subsection{Evolution of Anthocyanin and Phenolic Acid Standards in Simulated Gastrointestinal pH and Temperature Conditions}

Wine is a complex system that consists of multiple nutrients and components. To elucidate the relations and degradation mechanism between anthocyanins and phenolic acids in wine, we further conducted the same digestion study using anthocyanin and phenolic acid standards (Table 3).

Table 3. Degradation products from malvidin 3-O-glucoside standard under the simulated digestion process.

\begin{tabular}{cccc}
\hline Compound & $\lambda \max$ & Precursor Ion & Product Ion \\
\hline Syringic acid & 274,279 & 197 & 153 \\
Peonidin 3-O-glucoside * & - & 463 & 301 \\
Malvidin 3-O-glucoside * & 525 & 493 & 331 \\
Malvidin 3-O-glucoside chalcone * & 343 & 511 & 349,223 \\
Malvidin 3-O-glucoside chalcone & 343 & 509 & 347,221 \\
\hline
\end{tabular}

Note: * Positive electrospray ionization mode.

To ensure that phenolic acids are the products of the anthocyanins degradation during the digestion process, a commercial standard of malvidin 3-O-glucoside was incubated under the same digestive conditions, and its degradation products were identified using HPLC and UPLC-MS (Table 3 and Appendix A, Figure A1a,b). A tiny amount of peonidin 3-O-glucoside was present in the commercial malvidin 3-O-glucoside standard ethanol solution. After incubating the standard solution under the simulated digestion conditions, a compound with a precursor ion of $m / z 197$ and a product ion of $m / z 153$ was detected at extraction ion mode (Appendix A Figure A2a,b). This degradation product was identified as syringic acid. Besides, malvidin 3-O-glucoside chalcone was also found due to the presence of its precursor ion of $m / z 509$ and product ion of $m / z 347$ in negative electrospray ionization mode, and the presence of its precursor ion of $m / z 511$ and product ion of $m / z 349$ in positive electrospray ionization mode [59-61] (Appendix A Figure A2c and Table 3). Similarly, vanillic acid and protocatechuic acid were also identified as the malvidin 3-O-glucoside degradation products after the simulated digestion due to their retention time and maximal wavelength feature.

Along with the digestion process, the commercial standards showed the similar concentration during the simulated gastric digestion process. Syringic acid and protocatechuic acid were found with a low level in the solution during the simulated gastric digestion. The simulated intestinal digestion significantly decreased the concentration of the anthocyanins, which resulted in a dramatic concentration increase of syringic acid, protocatechuic acid, and vanillic acid (Figure 9a). Malvidin 3-O-glucoside chalcone exhibited a concentration decrease during the simulated gastric digestion process. During the simulated intestinal digestion, its concentration increased and then eventually decreased (Figure 9b). This indicated that chalcone conformation was an intermediate stage during the anthocyanins' degradation under the digestion process conditions [59]. It should be noted that the anthocyanin standard degradation percentage was not recovered by the formation of these phenolic acids, which was consistent with a previous report [51]. This indicated that further reactions might take place after the formation of phenolic acids. A further study needs to be conducted. Based on the structure of these three phenolics and the five basic anthocyanins (Figure 1), it can be deduced that protocatechuic acid, vanillic acid and syringic acid result from the B-ring of cyanidin 3-O-glucoside, peonidin 3-O-glucoside and malvidin 3-O-glucoside, respectively $[46,47,58]$. In this study, the commercial standards didn't contain cyanidin 3-O-glucoside. Thus, protocatechuic acid, 
the degradation product identified tentatively, might result from the other degradation pathway. However, this still needs to be further confirmed.

Additionally, we purified four anthocyanin standards from Yan 73 grape skin, including delphinidin 3-O-glucoside, petunidin 3-O-glucoside, malvidin 3-O-glucoside and peonidin 3-O-glucoside. Their purity was $94.7 \%, 98.1 \%, 94.3 \%$, and $99.0 \%$, respectively [62]. These anthocyanin standards in the wine model solution exhibited a concentration decrease after the incubation under the simulated gastric conditions $(13.10 \%, 20.81 \%, 35.10 \%$ and $6.25 \%$, respectively). The simulated intestinal conditions significantly decreased their concentration by $100 \%, 94.45 \%, 86.56 \%$, and $75.53 \%$, respectively (Figure 9c). These results indicated that peonidin 3-O-glucoside was the most stable monomeric anthocyanin, followed by malvidin 3-O-glucoside, petunidin 3-O-glucoside, and then delphinidin 3-O-glucoside. The evolution patterns of these standards were consistent with the evolution of these monomeric anthocyanins from the wine during the digestion process (Figure 4). It should be worth noting that a much higher degradation rate was observed in these monomeric anthocyanin standards compared to those in the wine, indicating that the wine matrix and/or other anthocyanin fractions in the wine might help protect monomeric anthocyanins from degradation under the digestion process conditions.

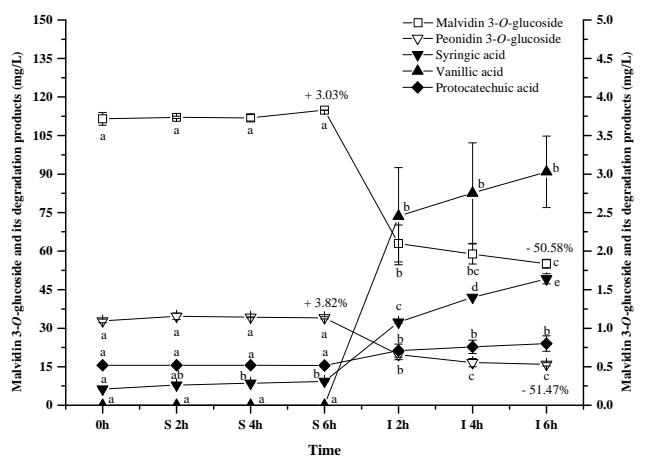

(a)

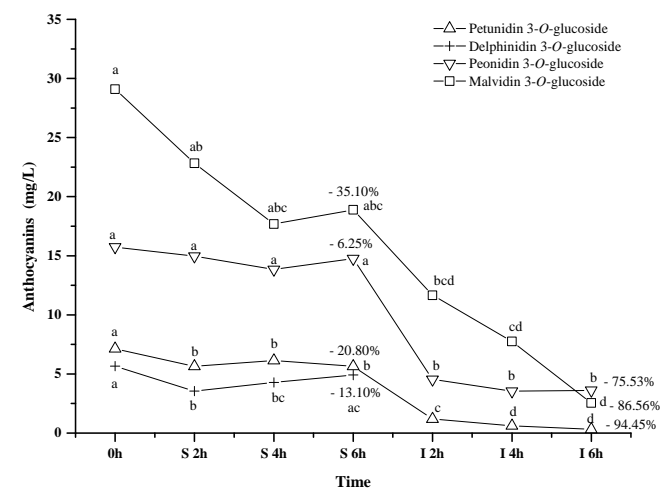

(c)

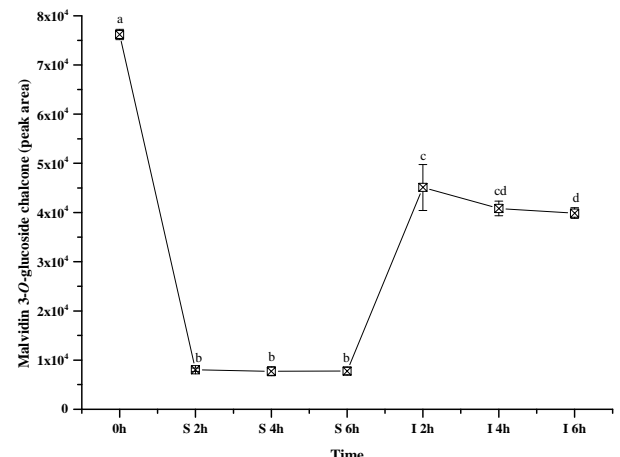

(b)

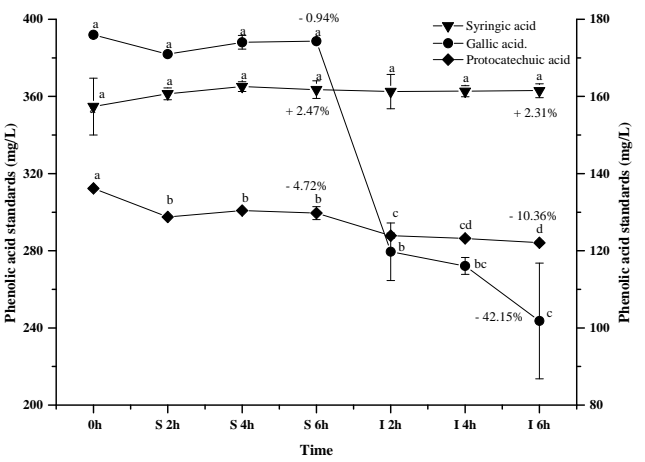

(d)

Figure 9. Content alteration of malvidin anthocyanin standard $(\mathbf{a}, \mathbf{c})$, its degradation products $(\mathbf{a}, \mathbf{b})$ and phenolic acid standards (d) during simulated digestion process ((a): malvidin 3-O-glucoside located at left axis, other compounds located at right axis; (b): the content of chalcone malvidin 3-O-glucoside was expressed as the peak area; (d): syringic acid located at left axis, protocatechuic acid and gallic acid located at right axis; wine sample: $\mathrm{W} 0$ h; S 2 h, S 4 h, S 6 h: samples under the gastric condition; I $2 \mathrm{~h}$, I $4 \mathrm{~h}$, I $6 \mathrm{~h}$ : samples under the intestinal condition; different lowercase letter means significant difference at 0.05 level). 
Syringic acid, protocatechuic acid, or gallic acid standard in the solution was incubated under the same simulated digestive condition to investigate their stability (Figure 9d). Syringic acid appeared to be stable to the digestive conditions. Similarly, the digestion process did not affect the stability of protocatechuic acid and its concentration only decreased by about $10 \%(p<0.05)$. Gallic acid remained stable under the simulated gastric conditions. However, a dramatic concentration decrease $(42.15 \%)$ happened to gallic acid in the simulated intestinal conditions. This result was identical with the previous report [63]. Under basic conditions, gallic acid might be changed into unstable quinone intermediates and/or other resonance forms which may ultimately oxidize in the presence of air to diketo derivatives or other degradation products [63]. However, the degradation mechanism is unclear at present.

It should be noted that these phenolic acids experienced the significant concentration decrease in the wine during the same simulated digestion process (Figure 8). This indicated that these phenolics (syringic acid, protocatechuic acid, or gallic acid) in the wine might further interact with other compounds (such as anthocyanin, quinone, chalcone, or alcohols), resulting in their concentration decrease $[43,51]$.

\section{Materials and Methods}

\subsection{Evolution of Anthocyanin and Phenolic Acid Standards in Simulated Gastrointestinal pH Conditions}

Four anthocyanin standards used for the gastrointestinal condition stability study, including delphinidin 3-O-glucoside, petunidin 3-O-glucoside, malvidin 3-O-glucoside, and peonidin 3-O-glucoside, were purified following a published method with some modifications [62]. Briefly, Yan 73 grape skin $(5 \mathrm{~g})$ was mixed with $1 \% \mathrm{HCl} v / v$ methanol $(20 \mathrm{~mL})$. The mixture was sonicated for $30 \mathrm{~min}$ at $40^{\circ} \mathrm{C}$, and then centrifuged at $8000 \mathrm{rpm} / \mathrm{min}$ for $5 \mathrm{~min}$ to collect the supernatant. The residue was then extracted using the same method two more times, and the supernatants were pooled and then filtered through a $0.45 \mu \mathrm{m}$ membrane filter. The resultant sample was purified using XAD-7HP resin (Amberlite, Sigma-Aldrich, Shanghai, China) and then each anthocyanin fraction was collected using a Shimadzu HPLC system (Shimadzu, Suzhou, China; pump: LC-6AD; diode array detector: SPD-M10-AVP; system controller: SCL-10AVP) through a fraction collector (FRC-10A). These four anthocyanins had a purity above $94 \%$, and were stored at $-20{ }^{\circ} \mathrm{C}$ before further study. A commercially available malvidin 3-O-glucoside standard (purity: $99.03 \%$ with peonidin-3-O-glucoside as impurity) was used to investigate the degradation products in the simulated gastrointestinal condition. Phenolic acid standards used for the digestion study included gallic acid, protocatechuic acid, vanillic acid, syringic acid, and $p$-coumaric acid, and these standards were purchased from Sigma-Aldrich.

\subsection{Chemicals}

Formic acid (HPLC grade) was purchased from Kemiou Chemical Reagent Co. Ltd. (Tianjin, China), whereas hydrochloric acid (analytical grade) was purchased from Xilong Chemical Industry Co. Ltd. (Chengdu, China). Ethanol, acetic acid, and sodium hydroxide were obtained from Kelong Chemical Reagents (Chengdu, China). Acetonitrile and methyl alcohol were of HPLC grade and purchased from Tedia Company Inc. (Shanghai, China).

\subsection{Grape Harvest and Wine-Making}

Ripen Cabernet Sauvignon (Vitis Vinifera L.) (total soluble solids: $21.5^{\circ}$ Brix; titrable acid: $7.6 \mathrm{~g} / \mathrm{L}$ ) grapes were harvested from the Caoxinzhuang vineyard (Yangling, China) in 2015 vintage. The harvested grapes were carefully handpicked and immediately transported back to our laboratory. The grape berries were manually de-stemmed and crushed into juice, and then thoroughly mixed with $50 \mathrm{mg} / \mathrm{L} \mathrm{SO}_{2}$ in a $10 \mathrm{~L}$ glass bottle. After $2 \mathrm{~h}, 20 \mathrm{mg} / \mathrm{L}$ commercial pectinase (Ex-color, Lallemand Inc., Aurillac, France) was added for $24 \mathrm{~h}$. Afterwards, $20 \mathrm{mg} / \mathrm{L}$ reactivated yeasts (RV002, Angel, Yichang, China) was inoculated into the juice to initiate wine alcoholic fermentation. During the fermentation 
process, temperature and must density were monitored three times daily. The fermentation was carried out on the skins at 25 to $28^{\circ} \mathrm{C}$ for 7 days. After the pomace was separated, the fermentation continued 2 days. After the alcoholic fermentation, $50 \mathrm{mg} / \mathrm{L} \mathrm{SO}_{2}$ was further mixed with the wine. The wine had a $12.0 \%$ alcohol level, $3.50 \mathrm{~g} / \mathrm{L}$ reducing sugar content, $6.20 \mathrm{~g} / \mathrm{L}$ acidity, and $0.48 \mathrm{~g} / \mathrm{L}$ volatile acidity.

\subsection{Digestion of Wine Sample, Anthocyanin Standards, and Phenolic Acid Standards in Simulated Gastrointestinal $\mathrm{pH}$ and Temperature Contidions}

Digestive enzymes were not included in the present simulated gastrointestinal condition since these enzymes have not been reported to digest phenolic compounds [64]. The wine sample was covered with tin foil to ensure dark conditions and was adjusted to $\mathrm{pH} 1.5 \mathrm{using} 0.1 \% \mathrm{HCl}$ solution. Then, the wine sample was incubated in a $37^{\circ} \mathrm{C}$ incubator for up to $6 \mathrm{~h}$. Afterwards, the resultant wine sample was immediately adjusted to $\mathrm{pH} 7.5$ using $1 \mathrm{M} \mathrm{NaOH}$ solution and incubated at $37^{\circ} \mathrm{C}$ for another $6 \mathrm{~h}$. At each time interval $(2 \mathrm{~h})$ during the period of $12 \mathrm{~h}$ under the simulated gastrointestinal conditions, each series of wine samples were prepared in duplicate for the analysis of anthocyanins and phenolic acids, and their corresponding marks were labelled W0 as origin wine sample, S $2 \mathrm{~h}$, $\mathrm{S} 4 \mathrm{~h}, \mathrm{~S} 6 \mathrm{~h}$ as treatments after 2, 4 and $6 \mathrm{~h}$ in simulated stomach conditions, I $2 \mathrm{~h}, \mathrm{I} 4 \mathrm{~h}$ and I $6 \mathrm{~h}$ as treatments after 2, 4, $6 \mathrm{~h}$ in simulated intestinal conditions.

Regarding anthocyanins or phenolic acid standards, each standard was initially dissolved in $12 \%$ ethanol solution at $\mathrm{pH}$ 3.5. The concentration of malvidin 3-O-glucoside standard was $111.48 \mathrm{mg} / \mathrm{L}$ with a $1.09 \mathrm{mg} / \mathrm{L}$ peonidin-3-O-glucoside concentration (impurity). The concentration of syringic acid, protocatechuic acid, and gallic acid were $354.78 \mathrm{mg} / \mathrm{L}, 136.16 \mathrm{mg} / \mathrm{L}$, and $175.97 \mathrm{mg} / \mathrm{L}$, respectively. The digestion of the standard solution followed the same procedure as the wine sample. Each series of these samples were also prepared in duplicate.

\subsection{Anthocyanins and Phenolic Acids HPLC Analyses}

Anthocyanins and their degradation products were directly analyzed using a Shimadzu LC-20AT HPLC instrument coupled with a photodiode array detector (Shimadzu, Suzhou, China). A Synergi Hydro-RP C18 column $(250 \times 4.6 \mathrm{~mm}, 4 \mu \mathrm{m}$, Phenomenex, Torrance, CA, USA) was used to separate the anthocyanins in the samples under a flow rate of $1 \mathrm{~mL} / \mathrm{min}$. The mobile phase consisted of (A) $2.5 \%$ formic acid $(v / v)$ in water: acetonitrile $(8: 1, v / v)$ and (B) $2.5 \%$ formic acid $(v / v)$ in water: acetonitrile $(4: 5, v / v)$. The gradient was programed as follows: 0 to $45 \mathrm{~min}, 0 \%$ to $35 \% \mathrm{~B} ; 45$ to $46 \mathrm{~min}, 35 \%$ to $100 \% \mathrm{~B} ; 46$ to $50 \mathrm{~min}, 100 \% \mathrm{~B}$ isocratic; 50 to $51 \mathrm{~min}, 100 \%$ to $0 \% \mathrm{~B}$; and 51 to $55 \mathrm{~min}, 0 \% \mathrm{~B}$ isocratic. The wavelength on the photodiode array detector was set at $520 \mathrm{~nm}$. Malvidin 3-O-glucoside was used as the external anthocyanin standard for the quantitation of all the anthocyanins in the wine.

Phenolic acids, including gallic acid, protocatechuic acid, vanillic acid, syringic acid, and $p$-coumaric acid were extracted according to a published method [65]. In brief, sample (1 mL) was mixed with ethyl acetate $(1 \mathrm{~mL})$ and acetonitrile $(0.5 \mathrm{~mL})$ in a $4-\mathrm{mL}$ centrifuge tube. The mixture was vortexed for $10 \mathrm{~s}$ and then centrifuged at $5400 \times g$ for $5 \mathrm{~min}$ to collect the organic phase. The sample was extracted two more times with the same extraction procedure. Afterwards, the organic phase extracts were combined and then evaporated under nitrogen stream at room temperature. The dryness was finally dissolved into $0.5 \mathrm{~mL}$ methanol for HPLC analysis. A Shimadzu HPLC, equipped with a Synergi Hydro-RP C18 column $(250 \times 4.6 \mathrm{~mm}, 4 \mu \mathrm{m}$, Phenomenex $)$, was used to analyze the phenolic acids under a flow rate of $1 \mathrm{~mL} / \mathrm{min}$. The mobile phase was comprised of (A) $0.1 \%$ acetic acid $(v / v)$ in water: acetonitrile $(8: 1, v / v)$ and (B) $0.1 \%$ acetic acid $(v / v)$ in water: acetonitrile $(4: 5, v / v)$. The gradient was as follows: 0 to $45 \mathrm{~min}, 0 \%$ to $35 \% \mathrm{~B} ; 45$ to $50 \mathrm{~min}, 35 \%$ to $100 \% \mathrm{~B} ; 50$ to $55 \mathrm{~min}, 100 \%$ B isocratic; 55 to $56 \mathrm{~min}, 100 \%$ to $0 \% \mathrm{~B}$; and 56 to $62 \mathrm{~min}, 0 \%$ B isocratic. Gallic acid, protocatechuic acid, vanillic acid, and syringic acid were detected at $280 \mathrm{~nm}$ on the photodiode array detector, whereas $p$-coumaric acid was measured at $320 \mathrm{~nm}$. These phenolic acids were quantified using their corresponding reference standard. 


\subsection{UPLC-ESI-MS/MS}

Anthocyanins and their degradation products after digestion in the simulated gastrointestinal tract were identified using a Waters Acquity UPLC system coupled with a BEH C18 column $(100 \times 2.1 \mathrm{~mm}$, $1.7 \mu \mathrm{m}$, Waters Corporation, Milford, MA, USA), a Waters 2489 UV-Visible detector, and a Synapt Q-TOF mass spectrometer (Waters Corporation). The column was maintained at $30{ }^{\circ} \mathrm{C}$ and the mobile phase consisted of (A) $5 \%$ formic acid $(v / v)$ in water and (B) $5 \%$ formic acid $(v / v)$ in methanol under a $0.3 \mathrm{~mL} / \mathrm{min}$ flow rate. The elution gradient was programed as follows: 0 to $30 \mathrm{~min}, 50 \% \mathrm{~B} ; 30$ to $35 \mathrm{~min}, 100 \% \mathrm{~B} ; 35$ to $37 \mathrm{~min}, 0 \% \mathrm{~B}$. A positive electrospray ion mode was used for the anthocyanins analysis, whereas phenolic acids was ionized under a negative mode with a $3.5 \mathrm{kV}$ capillary voltage, a $20 \mathrm{~V}$ cone voltage, and $100{ }^{\circ} \mathrm{C}$ dry temperature. A full scan mode from $m / z 50$ to 1500 was recorded. Masslynx 4.1 software (Waters Corporation) was used for data collection and analyses.

\subsection{Statistical Analysis}

Data were expressed as the mean \pm standard deviation of duplicate tests. The analysis of variance (ANOVA) was carried out using SPSS22.0 software (SPSS Inc., Chicago, IL, USA) under Tukey's honest significant difference at a significant level of $0.05(p<0.05)$.

\section{Conclusions}

In conclusion, most of anthocyanins in wine remained stable under simulated gastric conditions. However, they experienced a dramatic concentration decrease in a simulated intestinal digestion process. Their structure and matrix played an important role in their stability under digestive system conditions. Syringic acid, protocatechuic acid, and vanillic acid were found to result from anthocyanins' degradation and the anthocyanin chalcone was suggested to serve as an important degradation intermediate to form degradation products. Phenolic acid standards solution remained stable under simulated digestion process conditions. However, a dramatic decrease in their concentration happened to wine during the simulated digestion process.

Acknowledgments: This study was financially supported by the National Nature Science Foundation of China (Grant No. 31401479), National Key R\&D Program of China (Grant No. 2016YFD0400500), Key Research and Development Plan of Shaanxi Province (Grant Nos. 2017NY-184, 2017NY-144), and Scientific Research Project of Northwest A\&F University (Grant No. Z109021703).

Author Contributions: Hua Wang and Fuliang Han conceived and designed the experiments; Ping Yang and Chunlong Yuan performed the experiments; Yangie Liu, Lin Wang and Yang Liu analyzed the data; Fuliang Han wrote the manuscript.

Conflicts of Interest: The authors declare no conflict of interest.

\section{Appendix A}

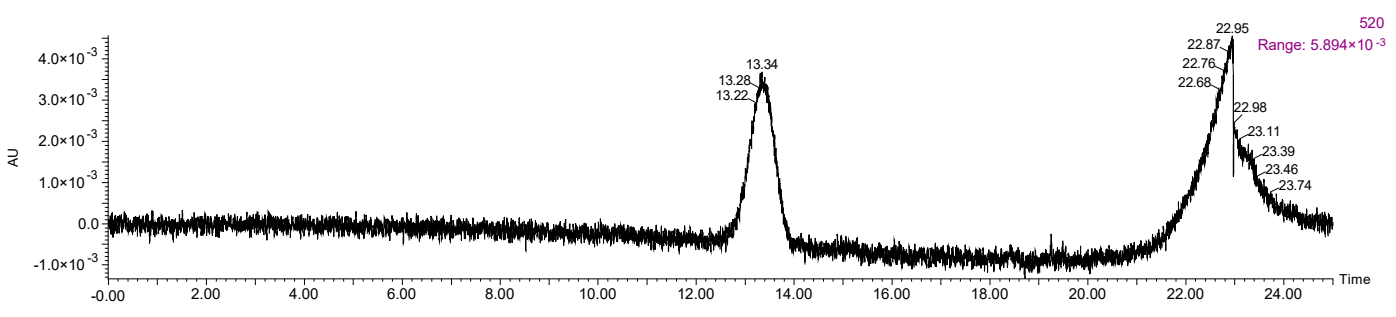

(a)

Figure A1. Cont. 


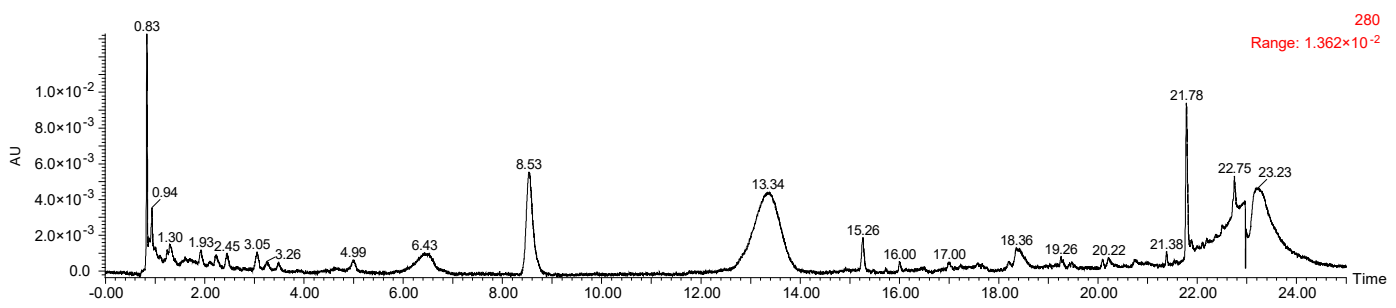

(b)

Figure A1. HPLC chromatography of (a) malvidin 3-O-glucoside standard at $520 \mathrm{~nm}$ and (b) its degradation products at $280 \mathrm{~nm}$ during the simulated digestion process.

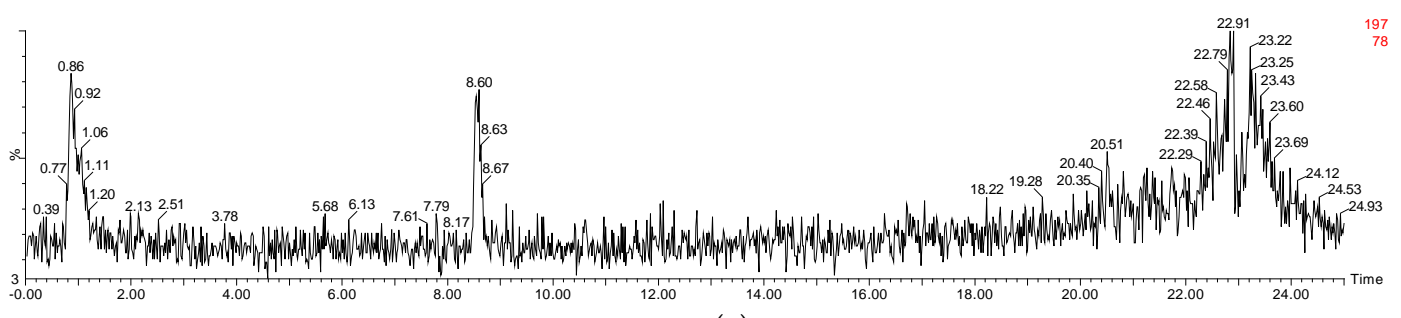

(a)

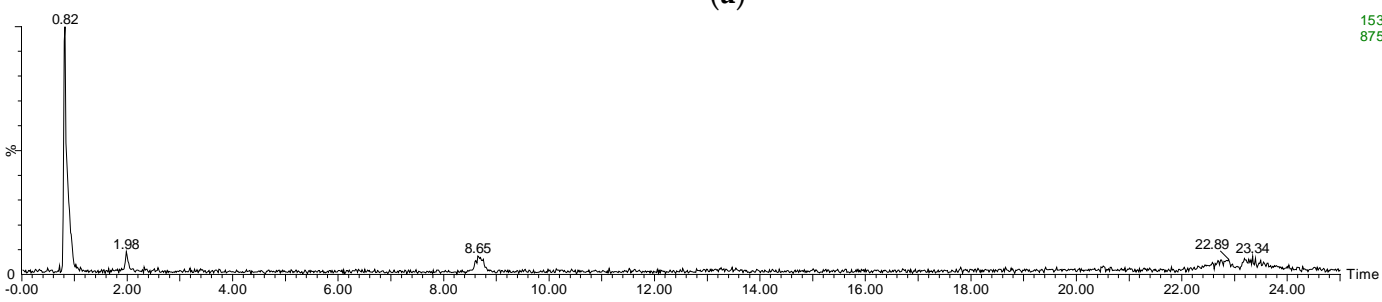

(b)

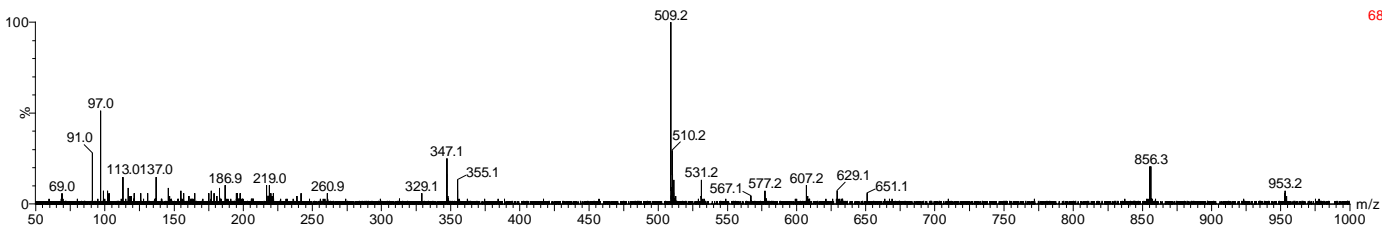

(c)

Figure A2. Mass spectrum of $(\mathbf{a}, \mathbf{b})$ syringic acid and ((c), negative electrospray ionization mode) malvidin 3-O-glucoside-chalcone during the simulated digestion process.

\section{References}

1. Han, F.L.; Li, Z.; Xu, Y. Contribution of monomeric anthocyanins to the color of young red wine: Statistical and experimental approaches. J. Food Sci. 2015, 80, C2751-C2758. [PubMed]

2. Han, F.L.; Zhang, W.N.; Pan, Q.H.; Zheng, C.R.; Chen, H.Y.; Duan, C.Q. Principal component regression analysis of the relation between CIELAB color and monomeric anthocyanins in young cabernet sauvignon wines. Molecules 2008, 13, 2859-2870. [CrossRef] [PubMed]

3. Baiano, A.; De Gianni, A.; Mentana, A.; Quinto, M.; Centonze, D.; Del Nobile, M.A. Colour-related phenolics, volatile composition, and sensory profile of Nero di Troia wines treated with oak chips or by micro-oxygenation. Eur. Food Res. Technol. 2016, 242, 1631-1646. [CrossRef]

4. Sáenz-Navajas, M.P.; Ferreira, V.; Dizy, M.; Fernández-Zurbano, P. Characterization of taste-active fractions in red wine combining HPLC fractionation, sensory analysis and ultra performance liquid chromatography coupled with mass spectrometry detection. Anal. Chim. Acta 2010, 673, 151-159. [CrossRef] [PubMed]

5. Ma, W.; Guo, A.Q.; Zhang, Y.L.; Wang, H.; Liu, Y.; Li, H. A review on astringency and bitterness perception of tannins in wine. Trends Food Sci. Technol. 2014, 40, 6-19. [CrossRef] 
6. Pojer, E.; Mattivi, F.; Dan, J.; Stockley, C.S. The case for anthocyanin consumption to promote human health: A review. Compr. Rev. Food Sci. Food Saf. 2013, 12, 483-508. [CrossRef]

7. Strathearn, K.E.; Yousef, G.G.; Grace, M.H.; Roy, S.L.; Tambe, M.A.; Ferruzzi, M.G.; Wu, Q.L.; Simon, J.E.; Lila, M.A.; Rochet, J.C. Neuroprotective effects of anthocyanin- and proanthocyanidin-rich extracts in cellular models of Parkinson's disease. Brain Res. 2014, 1555, 60-77. [CrossRef] [PubMed]

8. Rodriguez-Mateos, A.; Heiss, C.; Borges, G.; Crozier, A. Berry (Poly)phenols and cardiovascular health. J. Agric. Food Chem. 2014, 62, 3842-3851. [CrossRef] [PubMed]

9. Robertson, R.P. Red wine and diabetes health: Getting skin in the game. Diabetes 2014, 63, 31-38. [CrossRef] [PubMed]

10. Tsuda, T. Recent progress in anti-obesity and anti-diabetes effect of berries. Antioxidants 2016, 5, 13. [CrossRef] [PubMed]

11. Desjardins, J.; Tanabe, S.; Bergeron, C.; Gafner, S.; Grenier, D. Anthocyanin-rich black currant extract and cyanidin-3-O-glucoside have cytoprotective and anti-inflammatory properties. J. Med. Food 2012, 15, 1045-1050. [CrossRef] [PubMed]

12. Kuntz, S.; Kunz, C.; Rudloff, S. Inhibition of pancreatic cancer cell migration by plasma anthocyanins isolated from healthy volunteers receiving an anthocyanin-rich berry juice. Eur. J. Nutr. 2017, 56, 203-214. [CrossRef] [PubMed]

13. Basu, A.; Rhone, M.; Lyons, T.J. Berries: Emerging impact on cardiovascular health. Nutr. Rev. 2010, 68, 168-177. [CrossRef] [PubMed]

14. Cerletti, C.; Curtis, A.D.; Bracone, F.; Digesù, C.; Morganti, A.G.; Iacoviello, L.; de Gaetano, G.; Donati, M.B. Dietary anthocyanins and health: Data from FLORA and ATHENA EU projects. Br. J. Clin. Pharmacol. 2017, 83, 103-106. [CrossRef] [PubMed]

15. Li, D.; Zhang, Y.H.; Liu, Y.; Sun, R.F.; Xia, M. Purified anthocyanin supplementation reduces dyslipidemia, enhances antioxidant capacity, and prevents insulin resistance in diabetic patients. J. Nutr. 2015, 145, 742-748. [CrossRef] [PubMed]

16. Gholami, M. Biosynthesis of anthocyanins in shiraz grape berries. J. Biol. Chem. 2004, 640, 353-360. [CrossRef]

17. Kuhn, N.; Guan, L.; Dai, Z.W.; Wu, B.H.; Lauvergeat, V.; Gomès, E.; Li, S.H.; Godoy, F.; Arcejohnson, P.; Delrot, S. Berry ripening: Recently heard through the grapevine. J. Exp. Bot. 2013, 65, 4543-4559. [CrossRef] [PubMed]

18. Han, F.L.; Jiang, S.M.; He, J.J.; Pan, Q.H.; Duan, C.Q.; Zhang, M.X. Anthocyanins in 'Cabernet Gernischet' (Vitis vinifera L. Cv.) aged red wine and their color in aqueous solution analyzed by partial least square regression. Food Sci. Biotechnol. 2009, 18, 724-731.

19. Avizcuri, J.M.; Sáenz-Navajas, M.P.; Echávarri, J.F.; Ferreira, V.; Fernández-Zurbano, P. Evaluation of the impact of initial red wine composition on changes in color and anthocyanin content during bottle storage. Food Chem. 2016, 213, 123-134. [CrossRef] [PubMed]

20. Lago-Vanzela, E.S.; Rebello, L.; Ramos, A.M.; Stringheta, P.C.; Da-Silva, R.; García-Romero, E.; Gómez-Alonso, S.; Hermosín-Gutierrez, I. Chromatic characteristics and color-related phenolic composition of Brazilian young red wines made from the hybrid grape cultivar BRS Violeta ("BRS Rúbea" $\times$ "IAC 1398-21"). Food Res. Int. 2013, 54, 33-43. [CrossRef]

21. Dipalmo, T.; Crupi, P.; Pati, S.; Clodoveo, M.L.; Di Luccia, A. Studying the evolution of anthocyanin-derived pigments in a typical red wine of Southern Italy to assess its resistance to aging. LWT-Food Sci. Technol. 2016, 71, 1-9. [CrossRef]

22. Federico Casassa, L.; Bolcato, E.A.; Sari, S.E.; Fanzone, M.L.; Jofré, V.P. Combined effect of prefermentative cold soak and $\mathrm{SO}_{2}$ additions in Barbera D'Asti and Malbec wines: Anthocyanin composition, chromatic and sensory properties. LWT-Food Sci. Technol. 2016, 66, 134-142. [CrossRef]

23. Sivilotti, P.; Herrera, J.C.; Lisjak, K.; Česnik, H.B.; Sabbatini, P.; Peterlunger, E.; Castellarin, S.D. Impact of leaf removal, applied before and after flowering, on anthocyanin, tannin, and methoxypyrazine concentrations in 'Merlot' (Vitis vinifera L.) grapes and wines. J. Agric. Food Chem. 2016, 64, 4487-4496. [CrossRef] [PubMed]

24. Orduña, R.M.D. Climate change associated effects on grape and wine quality and production. Food Res. Int. 2010, 43, 1844-1855. [CrossRef] 
25. Cejudo-Bastante, M.J.; Rodríguez-Morgado, B.; Jara-Palacios, M.J.; Rivas-Gonzalo, J.C.; Parrado, J.; Heredia, F.J. Pre-fermentative addition of an enzymatic grape seed hydrolysate in warm climate winemaking. Effect on the differential colorimetry, copigmentation and polyphenolic profiles. Food Chem. 2016, 209, 348-357. [CrossRef] [PubMed]

26. Han, F.; Ju, Y.; Ruan, X.; Zhao, X.; Yue, X.; Zhuang, X.; Qin, M.; Fang, Y. Color, anthocyanin, and antioxidant characteristics of young wines produced from spine grapes (Vitis davidii Foex) in China. Food Nutr. Res. 2017, 61, 1339552. [CrossRef] [PubMed]

27. Sánchez-Ilárduya, M.B.; Sánchez-Fernández, C.; Viloria-Bernal, M.; López-Márquez, D.M.; Berrueta, L.Á.; Gallo, B.; Vicente, F. Mass spectrometry fragmentation pattern of coloured flavanol-anthocyanin and anthocyanin-flavanol derivatives in aged red wines of Rioja. Aust. J. Grape Wine Res. 2012, 18, 203-214. [CrossRef]

28. Oliveira, J.; Azevedo, J.; Silva, A.M.S.; Teixeira, N.; Cruz, L.; Mateus, N.; de Freitas, V. Pyranoanthocyanin dimers: A new family of turquoise blue anthocyanin-derived pigments found in port wine. J. Agric. Food Chem. 2010, 58, 5154-5159. [CrossRef] [PubMed]

29. De Freitas, V.; Mateus, N. Formation of pyranoanthocyanins in red wines: A new and diverse class of anthocyanin derivatives. Anal. Bioanal. Chem. 2011, 401, 1463-1473. [CrossRef] [PubMed]

30. Alberts, P.; Stander, M.A.; de Villiers, A. Advanced ultra high pressure liquid chromatography-tandem mass spectrometric methods for the screening of red wine anthocyanins and derived pigments. J. Chromatogr. A 2012, 1235, 92-102. [CrossRef] [PubMed]

31. Blanco-Vega, D.; Gómez-Alonso, S.; Hermosín-Gutiérrez, I. Identification, content and distribution of anthocyanins and low molecular weight anthocyanin-derived pigments in Spanish commercial red wines. Food Chem. 2014, 158, 449-458. [CrossRef] [PubMed]

32. Sousa, C.; Mateus, N.; Silva, A.M.S.; González-Paramás, A.M.; Santos-Buelga, C.; Freitas, V.D. Structural and chromatic characterization of a new malvidin 3-glucoside-vanillyl-catechin pigment. Food Chem. 2007, 102, 1344-1351. [CrossRef]

33. Pissarra, J.; Lourenço, S.; González-Paramás, A.M.; Mateus, N.; Buelga, C.S.; Silva, A.M.S.; Freitas, V.D. Structural characterization of new malvidin 3-glucoside-catechin aryl/alkyl-linked pigments. J. Agric. Food Chem. 2004, 52, 5519-5526. [CrossRef] [PubMed]

34. Marquez, A.; Serratosa, M.P.; Merida, J. Influence of bottle storage time on colour, phenolic composition and sensory properties of sweet red wines. Food Chem. 2014, 146, 507-514. [CrossRef] [PubMed]

35. Quaglieri, C.; Jourdes, M.; Waffo-Teguo, P.; Teissedre, P.L. Updated knowledge about pyranoanthocyanins: Impact of oxygen on their contents, and contribution in the winemaking process to overall wine color. Trends Food Sci. Technol. 2017, 67, 139-149. [CrossRef]

36. Garcia-Alonso, M.; Minihane, A.M.; Rimbach, G.; Rivas-Gonzalo, J.C.; de Pascual-Teresa, S. Red wine anthocyanins are rapidly absorbed in humans and affect monocyte chemoattractant protein 1 levels and antioxidant capacity of plasma. J. Nutr. Biochem. 2009, 20, 521-529. [CrossRef] [PubMed]

37. Fang, J. Bioavailability of anthocyanins. Drug Metab. Rev. 2014, 46, 508-520. [CrossRef] [PubMed]

38. Felgines, C.; Krisa, S.; Mauray, A.; Besson, C.; Lamaison, J.L.; Scalbert, A.; Merillon, J.M.; Texier, O. Radiolabelled cyanidin 3-O-glucoside is poorly absorbed in the mouse. Br. J. Nutr. 2010, 103, 1738-1745. [CrossRef] [PubMed]

39. Talavéra, S.; Felgines, C.; Texier, O.; Besson, C.; Manach, C.; Lamaison, J.L.; Rémésy, C. Anthocyanins are efficiently absorbed from the small intestine in rats. J. Nutr. 2004, 134, 2275-2279. [CrossRef] [PubMed]

40. He, J.; Wallace, T.C.; Keatley, K.E.; Failla, M.L.; Giusti, M.M. Stability of black raspberry anthocyanins in the digestive tract lumen and transport efficiency into gastric and small intestinal tissues in the rat. J. Agric. Food Chem. 2009, 57, 3141-3148. [CrossRef] [PubMed]

41. Borges, G.; Roowi, S.; Rouanet, J.M.; Duthie, G.G.; Lean, M.E.J.; Crozier, A. The bioavallability of raspberry anthocyanins and ellagitannins in rats. Mol. Nutr. Food Res. 2007, 51, 714-725. [CrossRef] [PubMed]

42. Stalmach, A.; Edwards, C.A.; Wightman, J.D.; Crozier, A. Gastrointestinal stability and bioavailability of (poly)phenolic compounds following ingestion of Concord grape juice by humans. Mol. Nutr. Food Res. 2012, 56, 497-509. [CrossRef] [PubMed]

43. Hanske, L.; Engst, W.; Loh, G.; Sczesny, S.; Blaut, M.; Braune, A. Contribution of gut bacteria to the metabolism of cyanidin 3-glucoside in human microbiota-associated rats. Br. J. Nutr. 2013, 109, 1433-1441. [CrossRef] [PubMed] 
44. Bermúdez-Soto, M.J.; Tomás-Barberán, F.A.; García-Conesa, M.T. Stability of polyphenols in chokeberry (Aronia melanocarpa) subjected to in vitro gastric and pancreatic digestion. Food Chem. 2007, 102, 865-874. [CrossRef]

45. Correa-Betanzo, J.; Allen-Vercoe, E.; McDonald, J.; Schroeter, K.; Corredig, M.; Paliyath, G. Stability and biological activity of wild blueberry (Vaccinium angustifolium) polyphenols during simulated in vitro gastrointestinal digestion. Food Chem. 2014, 165, 522-531. [CrossRef] [PubMed]

46. Fleschhut, J.; Kratzer, F.; Rechkemmer, G.; Kulling, S.E. Stability and biotransformation of various dietary anthocyanins in vitro. Eur. J. Nutr. 2006, 45, 7-18. [CrossRef] [PubMed]

47. Kay, C.D.; Kroon, P.A.; Cassidy, A. The bioactivity of dietary anthocyanins is likely to be mediated by their degradation products. Mol. Nutr. Food Res. 2009, 531, S92-S101. [CrossRef] [PubMed]

48. Aura, A.M.; Mattila, I.; Hyötyläinen, T.; Gopalacharyulu, P.; Cheynier, V.; Souquet, J.M.; Bes, M.; Le Bourvellec, C.; Guyot, S.; Orešič, M. Characterization of microbial metabolism of Syrah grape products in an in vitro colon model using targeted and non-targeted analytical approaches. Eur. J. Nutr. 2013, 52, 833-846. [CrossRef] [PubMed]

49. Março, P.H.; Scarminio, I.S. Q-mode curve resolution of UV-vis spectra for structural transformation studies of anthocyanins in acidic solutions. Anal. Chim. Acta 2007, 583, 138-146. [CrossRef] [PubMed]

50. Forester, S.C.; Waterhouse, A.L. Identification of cabernet sauvignon anthocyanin gut microflora metabolites. J. Agric. Food Chem. 2008, 56, 9299-9304. [CrossRef] [PubMed]

51. Keppler, K.; Humpf, H. Metabolism of anthocyanins and their phenolic degradation products by the intestinal microflora. Bioorgan. Med. Chem. 2005, 13, 5195-5205. [CrossRef] [PubMed]

52. Mosele, J.I.; Macià, A.; Romero, M.P.; Motilua, M.J.; Rubió, L. Application of in vitro gastrointestinal digestion and colonic fermentation models to pomegranate products (juice, pulp and peel extract) to study the stability and catabolism of phenolic compounds. J. Funct. Foods 2015, 14, 529-540. [CrossRef]

53. He, J.; Carvalho, A.R.; Mateus, N.; De, F.V. Spectral features and stability of oligomeric pyranoanthocyanin-flavanol pigments isolated from red wines. J. Agric. Food Chem. 2010, 58, 9249-9258. [CrossRef] [PubMed]

54. Woodward, G.; Kroon, P.; Cassidy, A.; Kay, C. Anthocyanin stability and recovery: Implications for the analysis of clinical and experimental samples. J. Agric. Food Chem. 2009, 57, 5271-5278. [CrossRef] [PubMed]

55. Kamonpatana, K.; Failla, M.L.; Kumar, P.S.; Giusti, M.M. Anthocyanin structure determines susceptibility to microbial degradation and bioavailability to the buccal mucosa. J. Agric. Food Chem. 2014, 62, 6903-6910. [CrossRef] [PubMed]

56. Escribano-Bailón, T.; Alvarez-García, M.; Rivas-Gonzalo, J.C.; Heredia, F.J.; Santos-Buelga, C. Color and stability of pigments derived from the acetaldehyde-mediated condensation between malvidin 3-O-glucoside and (+)-catechin. J. Agric. Food Chem. 2001, 49, 1213-1217. [CrossRef] [PubMed]

57. Santos-Buelga, C.; Bravo-Haro, S.; Rivas-Gonzalo, J.C. Interactions between catechin and malvidin-3-monoglucoside in model solutions. Zeitschrift für Lebensmittel-Untersuchung und Forschung 1995, 201, 269-274. [CrossRef]

58. Jiménez-Girón, A.; Queipo-Ortuño, M.I.; Boto-Ordóñez, M.; Muñoz-González, I.; Sánchez-Patán, F.; Monagas, M.; Martín-Álvarez, P.J.; Murri, M.; Tinahones, F.J.; Andrés-Lacueva, C.; et al. Moreno-Arribas, M.V. Comparative study of microbial-derived phenolic metabolites in human feces after intake of gin, red wine, and dealcoholized red wine. J. Agric. Food Chem. 2013, 61, 3909-3915. [CrossRef] [PubMed]

59. Dueñas, M.; Fulcrand, H.; Cheynier, V. Formation of anthocyanin-flavanol adducts in model solutions. Anal. Chim. Acta 2006, 563, 15-25. [CrossRef]

60. Zhao, M.; Luo, Y.; Li, Y.; Liu, X.; Wu, J.; Liao, X.; Chen, F. The identification of degradation products and degradation pathway of malvidin-3-glucoside and malvidin-3,5-diglucoside under microwave treatment. Food Chem. 2013, 141, 3260-3267. [CrossRef] [PubMed]

61. Lopes, P.; Richard, T.; Saucier, C.; Teissedre, P.L.; Monti, J.P.; Glories, Y. Anthocyanone a: A quinone methide derivative resulting from malvidin 3-O-glucoside degradation. J. Agric. Food Chem. 2007, 55, 2698-2704. [CrossRef] [PubMed]

62. Tang, K.; Li, Y.; Han, Y.; Han, F.; Li, J.; Nie, Y.; Xu, Y. Studies on the preparative isolation and stability of seven main anthocyanins from Yan 73 grape. J. Sci. Food Agric. 2014, 94, 2472-2481. [CrossRef] [PubMed]

63. Friedman, M.; Jürgens, H.S. Effect of $\mathrm{pH}$ on the stability of plant phenolic compounds. J. Agric. Food Chem. 2000, 48, 2101-2110. [CrossRef] [PubMed] 
64. Sun, J.H.; Lim, B.O.; Decker, E.A.; Mcclements, D.J. In vitro human digestion models for food applications. Food Chem. 2010, 125, 1-12.

65. Yang, P.; Li, H.Q.; Wang, H.; Han, F.L.; Jing, S.Y.; Yuan, C.L.; Guo, A.Q.; Zhang, Y.L.; Xu, Z.M. Dispersive Liquid-Liquid microextraction method for HPLC determination of phenolic compounds in wine. Food Anal. Method. 2017, 10, 2383-2397. [CrossRef]

Sample Availability: Samples of the compounds are not available from the authors.

(C) 2018 by the authors. Licensee MDPI, Basel, Switzerland. This article is an open access article distributed under the terms and conditions of the Creative Commons Attribution (CC BY) license (http:/ / creativecommons.org/licenses/by/4.0/). 